\title{
Linear Shear Viscoelasticity of Confined, End-Attached Polymers in a Near-Theta Solvent
}

\author{
LENORE L. DAI, ${ }^{1}$ STEVE GRANICK ${ }^{2-4}$ \\ ${ }^{1}$ Department of Chemical Engineering, Texas Tech University, Lubbock, Texas 79409 \\ ${ }^{2}$ Department of Materials Science and Engineering, University of Illinois, Urbana, Illinois 61801 \\ ${ }^{3}$ Department of Chemistry, University of Illinois, Urbana, Illinois 61801 \\ ${ }^{4}$ Department of Physics, University of Illinois, Urbana, Illinois 61801
}

Received 21 May 2005; revised 19 September 2005; accepted 19 September 2005 DOI: 10.1002 / polb.20659

Published online in Wiley InterScience (www.interscience.wiley.com).

\begin{abstract}
Diblock copolymers of polystyrene and polyvinylpyridine, end-attached to mica by the traditional method of selecting one block to be insoluble and the other block to be soluble in the solvent, were studied with surface-force experiments while immersed in trans-decalin, a near-theta solvent for the polystyrene block, with special attention given to the small-amplitude shear viscoelastic response. The relaxation time, defined as the inverse frequency at which the effective loss modulus equaled the effective storage modulus, was studied not only as a function of the film thickness but also as a function of the grafting density. The relaxation times started to slow in direct proportion to diminishing surface separation when the surface separation took the value $D \approx L_{\mathrm{o}} / 3$ (where $L_{\mathrm{o}}$ is the thickness of the uncompressed end-attached layer). Attempts to make comparisons with available theories met with limited success. To test experimentally the origin of this shear viscoelastic slowdown, similar measurements were made with adsorbed polystyrene with a molecular weight similar to that of the polystyrene moiety of the diblock copolymer, and it was found that high magnitudes of the effective viscoelastic shear moduli appeared only when the compression was much larger. In a control experiment in which interpenetration between opposed end-attached chains was precluded, we also studied the case of adsorbed polystyrene-polyvinylpyridine on one side and a bare mica surface on the other side, and the effective viscoelastic shear forces were reduced by nearly 1 order of magnitude. By inference, in the opposed diblock copolymer systems, we attributed the slowdown of the relaxation times with decreasing film thickness to the interpenetration of end-attached chains. Additional comments are made regarding the ratio of shear forces to compressive forces, which is called the small-strain friction coefficient. This is believed to be the first quantification of the linear-response relaxation time of end-attached polymer layers. $\odot 2005$ Wiley Periodicals, Inc. J Polym Sci Part B: Polym Phys 43: 3487-3496, 2005
\end{abstract}

Keywords: diblock copolymer; interfaces; mechanical properties; viscoelastic properties $\mathrm{edu})$

Correspondence to: S. Granick (E-mail: granick@mrl.uiuc.

Journal of Polymer Science: Part B: Polymer Physics, Vol. 43, 3487-3496 (2005)

(ㄷ) 2005 Wiley Periodicals, Inc. 


\section{INTRODUCTION}

When considering the forces that act between polymers at interfaces, we sometimes encounter the view that surface forces are necessarily at zero frequency, but this overlooks viscoelasticity. Thus, in addition to the well-documented problem of equilibrium forces and equilibrium conformations that are characteristic of polymers at interfaces, it is also relevant to inquire into characteristic timescales of motion and relaxation. In the spirit of classic studies of viscoelasticity, which took the approach of seeking to understand the response of the system to perturbations so small that they probed the dynamic structure of the system at rest, ${ }^{1}$ this laboratory has sought, in the field of surface forces, to measure the linear viscoelastic response.

Accounts of the linear viscoelastic response of various confined homopolymer systems have been described elsewhere. ${ }^{2-6}$ Here we consider endattached polymer chains. To assemble them, we adopt the method pioneered by Tirrell et al., ${ }^{7}$ in which diblock copolymers are end-attached to mica by the selection of one block to be insoluble and the other block to be soluble in the solvent of study.

For such systems of end-attached chains, a rather complete theoretical and experimental understanding has developed during the past 20 years of the equilibrium chain conformations and force-distance relations when the opposed layers are brought into proximity such that they become compressed. ${ }^{7-12}$ The characteristic feature is that these chains, which at a free solidliquid interface are stretched by the crowding of adjacent chains at the surface, become squeezed together. Under good solvent conditions, it appears that the interpenetration of the opposed layers is minimal even after the layers have been compressed to substantially less than their unperturbed thickness. ${ }^{8,9,12}$ In this study, we are interested, on the contrary, in the case in which the solvent quality is near-theta.

The surface-force apparatus, in which two molecularly thin polymer layers can be placed at controlled separations, presents an attractive method by which to approach this problem experimentally. One might consider seeking to obtain equivalent data from shearing block copolymer lamellae, but it is difficult to use a macroscopicsize system for this purpose because rheology is then prone to be dominated by defects within the block copolymer microstructure. Using ap- proaches derived from the surface-force apparatus, others have previously addressed polymer brushes in shear, ${ }^{13-15}$ but always when the strain, and the resulting shear rate, were high. This is the regime of kinetic friction, in which forces are rate-independent or (as we have found in this laboratory) nearly so, ${ }^{16}$ and the systems are far from equilibrium. In contrast, the experiments described here employed a modified surface-force apparatus ${ }^{17}$ in which the amplitude of oscillatory shear could be controlled to be as small as $<0.1 \AA$ to ensure a linear response.

To the best of our knowledge, a quantitative comparison of theoretical predictions of the linear viscoelastic response with experimental data in this type of system has not been attempted previously, apart from earlier communications of partial results ${ }^{18,19}$ and the partial report of some of these data in a Ph.D. thesis. ${ }^{20}$ Separately, the force-distance profiles of these same end-attached systems were described. ${ }^{21}$

\section{EXPERIMENTAL}

\section{Rheometer}

The modification of a surface-force apparatus to perform oscillatory shear measurements is described in detail elsewhere. ${ }^{3,17}$ Briefly, shear was induced by the application of sinusoidal voltage to a piezoelectric bimorph element on a device holding the upper surface. The ensuing lateral motion was resisted by the viscoelastic fluid layer confined between the two surfaces, and the complex shear force calculated from the damping and phase shift of the oscillation, as detected from the voltage induced in a second, symmetrically placed the bimorph element. The response was separated into an elastic force (in phase with the drive) and a dissipative force (out of phase). The elastic and viscous force constants, $g^{\prime} \equiv f_{\text {elastic }} / x$ and $g^{\prime \prime} \equiv f_{\text {viscous }} / x$ (where $x$ is the displacement amplitude in oscillatory shear deformation), were normalized by the effective contact area, $A_{\text {eff }}$, multiplied by the film thickness to give the effective elastic and viscous shear moduli, $G_{\text {eff }}^{\prime}$ and

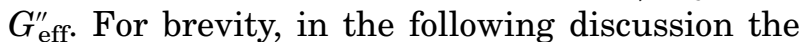
subscripts will be dropped when referring to these moduli. The contact area for the thinnest films was measured from the measured diameter of the flattened tip of the interference fringes, as the interferences fringes visibly deformed in this regime of high compression. For thicker films, $A_{\text {eff }}$ 
Table 1. Characteristics of the PS/PVP Diblock Copolymers

\begin{tabular}{|c|c|c|c|c|c|c|c|}
\hline Code & $\begin{array}{r}M_{\mathrm{n}} \text { for } \mathrm{PS} \\
\left(\mathrm{g} \mathrm{mol}{ }^{-1}\right)\end{array}$ & $\begin{array}{l}M_{\mathrm{n}} \text { for PVP } \\
\left(\mathrm{g} \mathrm{mol}^{-1}\right)\end{array}$ & $M_{\mathrm{w}} / M_{\mathrm{n}}$ & $\begin{array}{l}R_{\mathrm{G}} \text { for } \\
\text { PS }(\AA)^{\mathrm{c}}\end{array}$ & $\begin{array}{r}L_{o, d r y} \\
(\AA)^{\mathrm{d}}\end{array}$ & $\begin{array}{c}\sigma_{\mathrm{dry}} \\
\left(\text { chains } / \mathrm{m}^{2}\right)^{\mathrm{e}}\end{array}$ & $\begin{array}{c}\text { Graft } \\
\text { Spacing }(\AA)^{\mathrm{e}}\end{array}$ \\
\hline Polymer $A^{a}$ & 32,300 & 32,700 & 1.05 & 51 & 43 & $1.3 \times 10^{16}$ & 87 \\
\hline Polymer $\mathrm{B}^{\mathrm{b}}$ & 52,500 & 28,100 & 1.07 & 65 & 48 & $1.1 \times 10^{16}$ & 95 \\
\hline Polymer $\mathrm{C}^{\mathrm{b}}$ & 55,400 & 9,200 & 1.03 & 67 & 27 & $3.4 \times 10^{16}$ & 54 \\
\hline
\end{tabular}

a Donated by Professor Hiroshi Watanabe of Kyoto University (Japan).

${ }^{\mathrm{b}}$ Purchased from Polymer Sources, Inc. (Québec, Canada).

${ }^{\mathrm{c}}$ Calculated from the molecular weight.

${ }^{\mathrm{d}}$ Measured thickness of dry layers.

e Calculated from the dry thickness under the assumption of the same density as that for bulk PS and PVP.

was estimated from the film thickness, $D$, with the Langbein approximation, $A_{\text {eff }} \approx 2 \pi R D$, where $R$ is the mean radius of curvature of the mica surfaces and $D$ is their separation. Experimental evidence that supports the normalization of shear forces according to the Langbein estimate of $A_{\text {eff }}$ has been presented previously. ${ }^{4}$

Recently, the influence was reported on surface-force experiments of platinum nanoparticles produced by the standard method of cutting mica sheets with a hot platinum wire. ${ }^{22,23}$ This is not believed to influence the findings presented here because the mica surfaces went through several steps of solvent immersion and were covered with a dense polyvinylpyridine (PVP) layer. Even more important, with respect to the reported particle height $(\sim 2.3 \mathrm{~nm})$, the surface separations of interest here were considerably larger.

The quoted surface separations discussed here $(D)$ refer to the separation of two polystyrene (PS) layers with the thickness of dry PVP $(<1 \mathrm{~nm})$ subtracted. The shear linearity was verified by the variation of the shear amplitude to ensure that the output was proportional to the input. Note also that for the end-attached chains, the shear dynamic measurements were repeatable and showed no history dependence when the layers were compressed, separated, and compressed again.

\section{Samples}

We began with a polymer, polymer A (see Table 1), generously donated by Professor Hiroshi Watanabe of Kyoto University. Seeking to obtain higher grafting densities, later we purchased polymers B and C from Polymer Sources, Inc. (Québec, Canada). All these polymer samples were used as received. trans-Decalin (trans-decahydronaphthalene; purity $=99 \%$ ) was purchased from Fluka. Toluene (purity $>99.8 \%$, high-performance-liquid-chromatography-grade) was purchased from Sigma-Aldrich. These solvents were stored before use over molecular sieves (5 Aldrich) to remove trace moisture. In addition, the solvents were filtered just before use through $0.5 \mu \mathrm{m}$ Millex-SR filters. The PS-PVP solutions were prepared in toluene below the critical micelle concentration at concentrations of 5 $10 \mu \mathrm{g} \mathrm{mL} \mathrm{m}^{-1}$, as it is known that the amount adsorbed is insensitive to the concentration in this range of concentrations. ${ }^{24}$ The solutions were mixed at least $24 \mathrm{~h}$ before the adsorption process to ensure complete dissolution. Characteristics of the PS-PVP diblock copolymers are listed in Table 1, which lists not only the block molecular weights and polydispersities but also the dry thicknesses and grafting densities.

\section{Surface-Coating Methods}

For most experiments, the bare mica sheets were first glued onto silica lenses with a 50:50 mixture by weight of dextrose and galactose. The thickness of the bare mica sheets was calibrated in the surface-force apparatus, and then the sheets were immersed in a polymer solution for $2 \mathrm{~h}$. In experiments involving asymmetric brush layers (polymer on one side and bare mica on the other), the mica thickness was calibrated after the experiment with different sheets of mica that were cleaved at the same time and had identical thicknesses. After adsorption, the surfaces were soaked in pure toluene for another $2 \mathrm{~h}$. The preparations were performed within small glass vials outside the surface-force apparatus. After these adsorption and washing procedures, the coated surfaces were mounted within the surface-force apparatus. Toluene evaporated rapidly. Then, a droplet of trans-decalin was introduced between the sur- 
faces with a pipette. The inside environment of the apparatus was kept dry by a hygroscopic chemical, $\mathrm{P}_{2} \mathrm{O}_{5}$ powder, and was kept saturated with solvent vapor by the addition of droplets of trans-decalin to the bottom of the sample chamber.

One tricky point was the need to control the water content of the solvent. Unless the solvent was especially dried by molecular sieves as described previously, it seemed that moisture competed with PVP for adsorption. When the precaution was not taken to dry the solvents in this fashion, the surfaces began to repel one another, starting at the unreasonably large separation of several micrometers, and visible black spots were seen under an optical microscope.

\section{Quantification of the Amount Adsorbed}

After the experiments were completed, the polymer-laden surfaces were removed from the apparatus, rinsed with pure toluene to wash off transdecalin, and then dried under flowing argon for at least $3 \mathrm{~h}$. The thickness of the PS-PVP dry layer was measured by multiple-beam interferometry after the remounting of the surfaces into the surface-force apparatus. Knowing the thickness of the dry diblock layers and assuming the same densities of PVP and PS as those in the bulk, we calculated the grafting density.

\section{RESULTS}

In this section of the article, our main concern is first to quantify what determined the relaxation times of these viscoelastic thin films and next to determine how the linear shear viscoelastic spectra of these confined systems correlated to the normal load that squeezed them together. The plan of presentation is as follows. We begin by describing behavior for one system and contrast it with two other systems in which the grafting density was less. Then, to determine how essential to the results it was to have systems consisting of end-attached chains, we contrast findings with end-attached chains to similar experiments with homopolymer chains of the same chemical makeup but adsorbed statistically rather than by end attachment. Finally, to determine how essential to the results it was to have the symmetric situation of opposed end-attached polymer layers, we contrast findings with the asymmetric situation in which an end-attached polymer layer was pressed against a bare, uncoated mica surface.
We begin with two comments about how these experiments were interpreted. In the first place, there is the question of how to know the solventquality conditions for polymers that reside in a thin film, as these might differ from those for these same polymers in the bulk. ${ }^{25}$ We believe that because the bulk theta temperature of PS in trans-decalin is $20^{\circ} \mathrm{C}$, it is reasonable to interpret these thin-film results from this perspective, but readers should keep in mind that even if this were untrue, it would not change the data, and this is the main point of this article. Although it is valid to consider the possibility of confinementinduced shifts, to do so would go beyond the scope of this study and at any rate would not alter the experimental findings that compose the main point of this article.

Second, we believe that these rheological experiments on end-attached diblock copolymers were dominated by the scenario in which two polymer brushes rubbed against each another. It is true that although the PS block was close to theta-solvent conditions, this was not so for the PVP block, as PVP is insoluble in the solvent that we studied. When one considers that the dry thickness of the PVP layers was less than $1 \mathrm{~nm}$ and that the PS moieties swelled under these solvent conditions, it is not reasonable to expect that much of the dissipation would occur within the PVP portion of these diblock copolymers. To put this supposition to a factual test, the first test should be force-distance relations when these layers are compressed, but a survey of the literature shows that for this property, the force-distance relations can be understood without regard to the PVP layer, which is thin because the solvent environment is poor. ${ }^{12}$ The same should be so of shear viscoelastic responses. Further support for this comes when one recalls that the compressive-force/distance relations of these systems are known to be reversible.

\section{Highest Grafting Density}

We begin with the diblock copolymer that adsorbed most densely, polymer C. As a function of the film thickness, Figure 1 compares the compressive load with the viscous and elastic shear forces at $1 \mathrm{~Hz}$ and $0.5 \mathrm{~nm}$ shear deformation. The onset of shear forces coincided with the application of a normal load.

Although these data in units of force give one a feeling for the magnitudes of the raw data, normalization was necessary for the most useful 


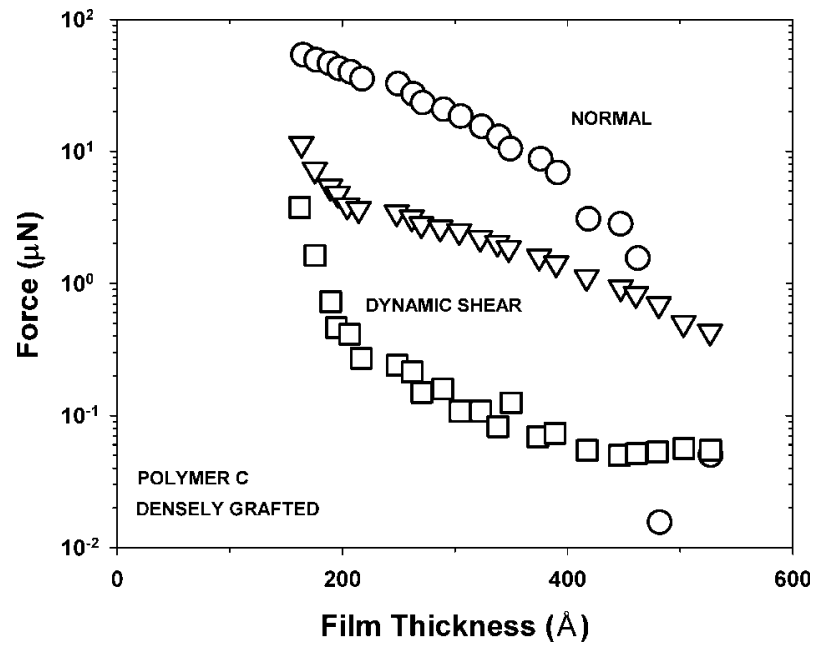

Figure 1. Force-distance profiles measured when two brush layers of polymer C (highest grafting density) were compressed to different surface-surface spacings while immersed in trans-decalin at $24{ }^{\circ} \mathrm{C}$. The forces are plotted against the surface separation. The circles denote the normal repulsive forces. The dynamic shear forces measured at $1 \mathrm{~Hz}$ are elastic (triangles) or viscous (squares) for a shear displacement of $5 \AA$. No dynamic shear forces were large enough to be detected until the onset of repulsion $(\approx 580 \AA)$.

quantitative comparison. With the normal load kept constant, the effective viscous and elastic shear moduli, $G^{\prime \prime}(\omega)$ and $G^{\prime}(\omega)$, respectively, were measured as a function of the angular frequency, $\omega$. When these layers were compressed to be less than $250 \AA$ thick, a dependence on the frequency was observed. Figure 2 shows frequency spectra measured at three plate separations of less than $200 \AA$; other similar data were also taken at other separations but for brevity are not shown. The magnitudes of the viscoelastic moduli grew as the layers were squeezed together. In the analysis presented next, the relaxation time is defined as the inverse frequency $(2 \pi / \omega)$ at which $G^{\prime \prime}(\omega)$ is equal to $G^{\prime}(\omega)$.

\section{Relaxation Times for All Three End-Attached Systems}

Similar experiments performed to investigate two systems that adsorbed less densely (see Table 1) also showed how their viscoelastic spectra shifted in magnitude and frequency as they were compressed. From data similar to those illustrated in Figure 2, relaxation times were also defined, just as was done for the layers that adsorbed most densely. In the manner described in the Discussion section, these times were normalized for changes in the monomeric friction coefficient.

The dependence of the normalized relaxation time, $\tau$, on the film thickness, shown in Figure 3, shows the same linear trend for all three systems but at well-separated distances that depended on the system at hand. It is striking to notice, in all three cases, a linear decrease of the relaxation time as the plate separation decreased. Extrapolating to $\tau \rightarrow 0$, one finds that the intercept was always less than the thickness, $L_{0}$, of one unperturbed brush layer. Specifically, the extrapolated thickness was $D \approx 0.7 L_{\mathrm{o}}$ in all three systems, that is, the compression of each of the opposed brushes to approximately one-third of its unperturbed thickness.

\section{Comparison with the Adsorbed Homopolymer}

A comparison was made with PS homopolymer. In this case, the chains were surface-attached solely by weak statistical adsorption of the segments along the PS chain, so the expected chain conformations were very different. ${ }^{21} \mathrm{~A}$ homopolymer of PS was selected with a weight-average molecular weight $\left(M_{\mathrm{w}}\right)$ of $37,900 \mathrm{~g} \mathrm{~mol}^{-1}$ [weight-average molecular weight/number-average molecular weight $\left(M_{\mathrm{w}} / M_{\mathrm{n}}\right)=1.02$; Toyo-Soda]. It was

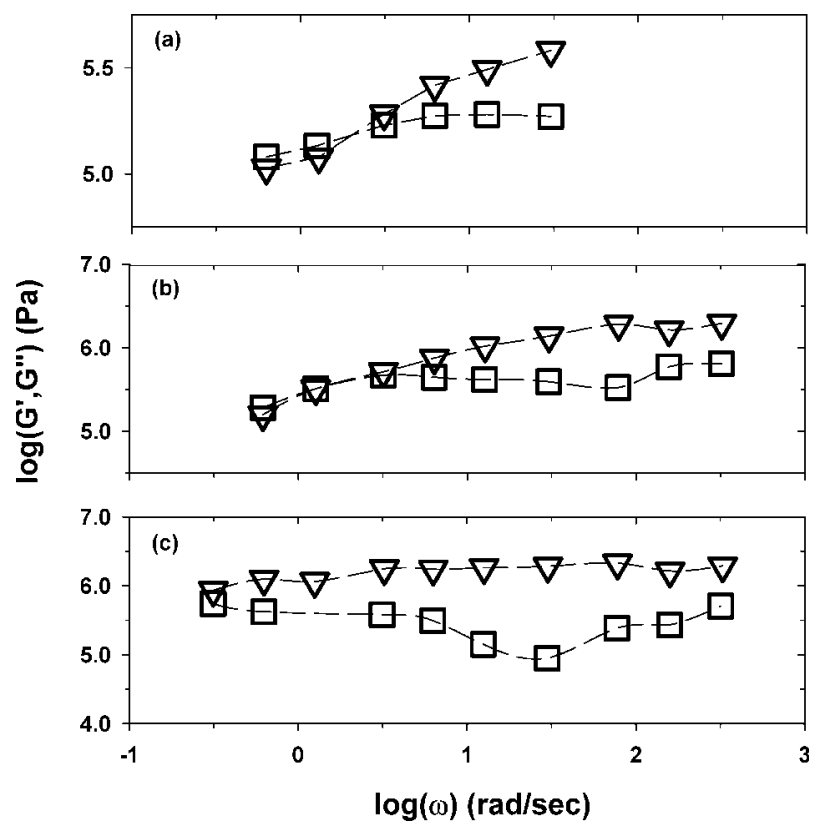

Figure 2. $(\nabla)$ Effective storage shear modulus, $G^{\prime}(\omega)$, and $(\square)$ effective loss shear modulus, $G^{\prime \prime}(\omega)$, plotted against $\omega$ on log-log scales for opposed layers of polymer $\mathrm{C}$ at three different surface separations: (a) $D=190 \AA$, (b) $D=170 \AA$, and (c) $D=160 \AA$. 


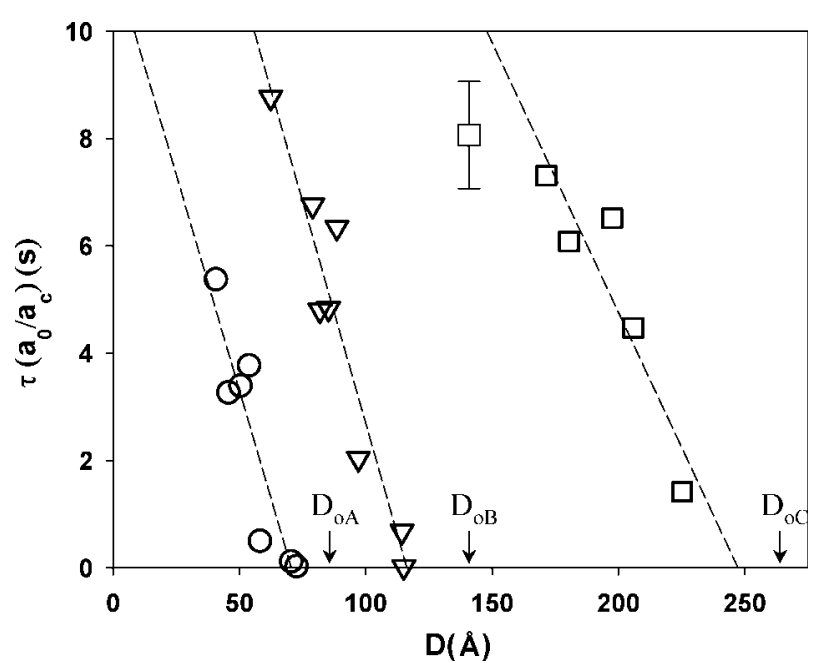

Figure 3. Thickness dependence of the long shear relaxation time, $\tau$, after normalization for differences of the monomeric friction coefficient (here the shift factor $a_{\mathrm{o}} / a_{\mathrm{c}}$ denotes the normalization), for the three tethered chain systems: $(\bigcirc)$ polymer A, $(\nabla)$ polymer $\mathrm{B}$, and ( $\square$ ) polymer C. The vertical arrows labeled $D_{\mathrm{oA}}, D_{\mathrm{oB}}$, and $D_{\mathrm{oC}}$ show the thickness of a single layer of the respective end-attached polymer $(\mathrm{A}, \mathrm{B}$, and $\mathrm{C}$, as specified in Table 1). This is defined as one-half of the film thickness at which repulsive normal forces were first observed to oppose compression of these opposed layers. Bearing in mind that relaxation times slow with increasing polymer concentration even in bulk solutions, to isolate the effects of confinement, we normalized these relaxation times with the Williams-Landel-Ferry equation ${ }^{1,20}$ by the shift factor $a_{\mathrm{o}} / a_{\mathrm{c}}$ to the mean PS concentration of the overlapping end-attached chains corresponding to the surface separation at which shear forces were first detected.

allowed to adsorb onto mica from a solution with a concentration of $1 \mathrm{mg} \mathrm{mL}^{-1}$ in toluene.

Figure 4 shows the shear-force/separationforce profile at $1 \mathrm{~Hz}$ for opposed layers; the compressive-force/distance profile is included for comparison. Forces of substantial magnitude appeared only when the compression was much larger than for the diblock copolymer system in Figure 1, by more than an order of magnitude. Normalization (not shown) to express these data in the language of $G^{\prime}(\omega)$ and $G^{\prime \prime}(\omega)$ gave the same conclusion.

As for any other experimental measurement, the data presented here carry a certain uncertainty. It is worthwhile to note that the signal-tonoise ratio in all of these figures is typical of dynamic surface-force experiments, except for the viscous forces shown in Figure 4. The significant scatter in the viscous forces shown in Figure 4 is not understood, but the main point is that the elastic shear component always dominated.

\section{Comparison with Polymer Brush in Contact with Bare Mica}

The data presented thus far suggested to us the hypothesis that the higher shear forces observed in the diblock copolymer systems might reflect interdigitation of the opposed end-tethered chains. To explore this hypothesis, experiments were performed in which the diblock copolymer was allowed to adsorb to one mica surface and then was exposed to bare mica on the other side. This precluded the possibility that shear forces would originate from opposed, interdigitated layers.

Figure 5, the analogue for this system of Figures 1 and 4, shows force-separation profiles. As this contained one sole end-attached layer, it is pleasing to see that compressive forces were observed at separations half as large as those in Figure 1. More significantly, the shear forces were uniformly lower than those for the brush-brush case. In the discussion that follows, we interpret this finding as confirming the hypothesis.

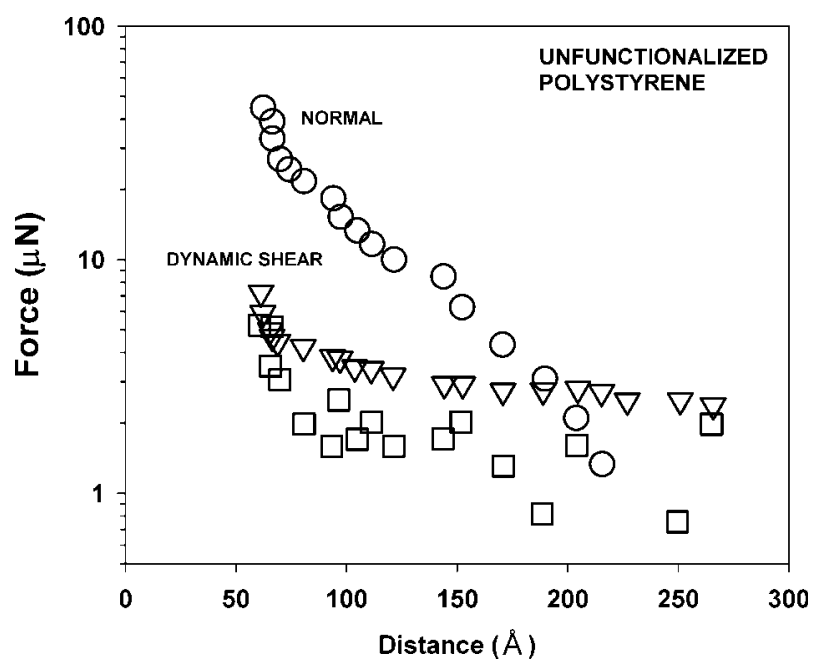

Figure 4. Force-distance profiles measured when two layers of adsorbed PS homopolymer with $M_{\mathrm{w}}=37,900 \mathrm{~g}$ $\mathrm{mol}^{-1}$ were compressed to variable spacing in the same solvent and at the same temperature used for the preceding figures (trans-decalin at $24^{\circ} \mathrm{C}$ ). The force is plotted against the film thickness. Circles denote the normal force. The dynamic shear forces at $1 \mathrm{~Hz}$ are elastic (triangles) or viscous (squares) for a shear displacement of $5 \AA$. The significant scattering of the viscous forces in this figure is not understood, but the main point is that the elastic shear component always dominated, regardless of the scatter in the viscous component. 


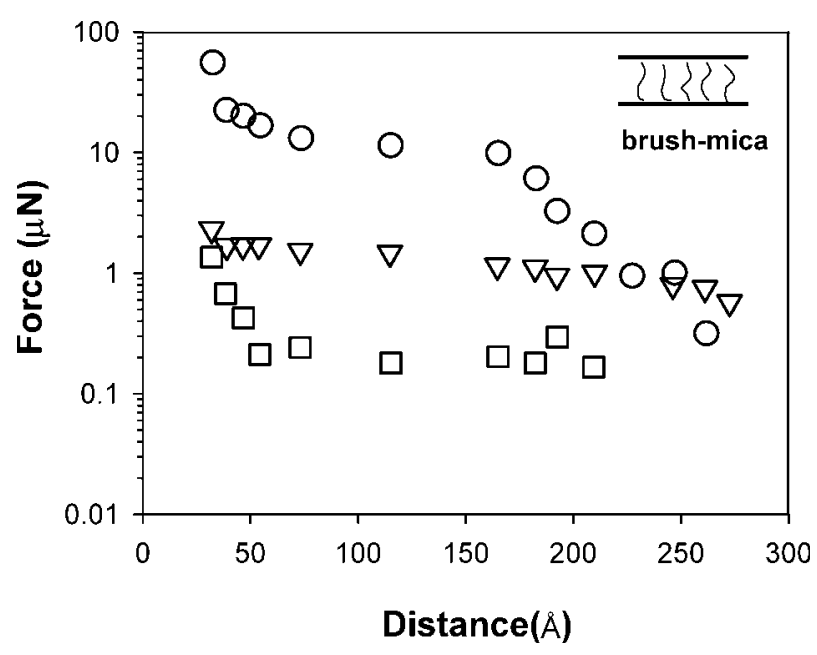

Figure 5. Force-distance profiles for a layer of polymer $\mathrm{C}$ on one side and a layer of bare mica on the other. Normal compressive forces are denoted by circles. Dynamic shear forces at $1 \mathrm{~Hz}$ are elastic (triangles) or viscous (squares) at $1 \mathrm{~Hz}$ for a shear displacement of $5 \AA$. The distance scale for the brushmica case is half of that for the brush-brush case.

\section{DISCUSSION}

\section{Relaxation Times}

Although the data in earlier figures show that the relaxation times slowed with diminishing surface separation, one can imagine the trivial reason that relaxation might slow simply because the polymer segment concentration increased with diminishing plate separation, bringing the local polymer concentration closer to the glass-transition temperature $\left(T_{\mathrm{g}}\right)$. How much this might influence the findings can be estimated if we consider the mean concentration of polymer (PS) segments within the confined space. This follows simply from knowing the thickness of the dry layer (Table 1) and recognizing that the segment density within end-attached chains, whose idealized limit is a polymer brush, is essentially the same at every distance from the solid surface. To quantify the influence on the relaxation time, the influence on the monomeric friction coefficient was estimated with the method of Ferry. ${ }^{1}$ Although one can be sure that proximity to $T_{\mathrm{g}}$ must slow down relaxation when PS surface layers are compressed strongly, the upshot of this calculation was that the tendency included a minor correction, as the volume concentration of PS chains was always less than $\sim 40 \%$ within the range of film thicknesses from which relaxation times were quanti- fied. ${ }^{20}$ Nonetheless, for thoroughness, this correction was made for the data presented in Figure 3.

Many attempts were made to find ways to normalize the data in Figure 3 with a variable that would collapse the data as effectively as the empirical variable $D / L_{\mathrm{o}}$ indicated in this figure. For example, $\tau$ was plotted as a function of the concentration $\left(\phi_{\mathrm{PS}}\right)$, the fractional compression $\left(D / R_{\mathrm{G}}\right)$, and $D / R_{\mathrm{G}}{ }^{2}$. Here, $R_{\mathrm{G}}$ is the unperturbed radius of gyration. However, these attempts to collapse the data were unsuccessful.

We now compare the data about the relaxation time to available theories. To describe the linear dynamics of polymer brushes, a scaling description was first proposed by Halperin and Alexander. ${ }^{26}$ By considering the presumed reptation nature of polymer mobility when chains are entangled, they concluded that the time $\tau$ of a chain consisting of $N$ monomers scales as $\tau \sim N^{2}$ rather than $N^{3}$ expected from center-of-mass diffusion motion. Calculations of some global dynamic characteristics have also been performed with computer simulations. ${ }^{27-31}$ The translational and rotational motions of different chain segments and correlation functions of chain radii were calculated. The relaxation function of the total chain configuration and the time dependence of mean-square displacements have been discussed with the bond fluctuation model. ${ }^{28,29}$ This problem was also modeled by Joanny, ${ }^{32}$ Rubinstein and Obukhov, ${ }^{33}$ Klein et al., ${ }^{34}$ McLeish et al., ${ }^{35}$ and Semenov and coworkers. ${ }^{36,37}$ These diverse theories were often based on models in which the longest relaxation times of brushes proceed by mechanisms analogous to arm retraction when star-shaped polymers relax in the bulk.

We sought to interpret the magnitudes of the relaxation times using the chain-retraction mechanism for entangled star polymers. ${ }^{33}$ According to the expectation that the reptation time of chains increases exponentially with the free chain concentration in semidilute solution, ${ }^{38}$ it follows, for entangled polymer brushes in a theta solvent, that

$$
\tau \sim D^{-3} \exp (N D / 2 \Gamma)^{-2}
$$

where $\Gamma$ is the adsorbed amount of polymer in units of monomer mass per unit of area, $N$ is the degree of polymerization, and $D$ is the film thickness. However, the data are not consistent with the predicted power law in $D$ and the exponential relation in eq 1.

What can we conclude from the observation that the measured relaxation times did not 
appear to agree with predictions from the available models for end-attached chains? Apparently, the experimental system was unable to make a successful comparison with these models. We speculate that whereas the available models concern systems in which chain segments possess absolutely no enthalpic attraction for the surface, in our systems the segments did adsorb weakly. To put this into perspective, it has been shown that if segment-surface attraction is weaker than the end-group sticking energy, chains nonetheless stretch if the graft density is sufficiently dense, ${ }^{39,40}$ as also observed here. In this sense, we may consider that weak sticking does not appear to invalidate predictions regarding mean chain conformations. However, the influence on shear relaxation times appears to be major.

\section{How Shear Forces Depended on the Grafting Density}

Although we do not understand the phenomenon, a striking and reproducible aspect of these experiments was that although resistance to shear was observed for the case of highest grafting density starting at the onset of compressive forces (Fig. 1), this was not so for the cases of lower grafting density, in which case no shear force could be detected until $D<L_{\mathrm{o}}$. Moreover, the shear forces observed for the case of highest grafting density were more elastic than viscous, as can be observed in Figure 1. Further investigation (not shown) showed them to be frequency-independent. To quantify differences between these systems, the effective storage modulus, which has the units of energy per volume, was expressed as energy stored in units of $k_{\mathrm{B}} T$ per chain (where $k_{\mathrm{B}}$ is the Boltzmann constant and $T$ is the absolute temperature). The comparison presented here concerns measurements made at $1 \mathrm{~Hz}$ because at this frequency the most extensive data were available, but similar findings were obtained when the comparison was made at 5 and $52 \mathrm{~Hz}$. In Figure 6, the energy stored per chain is plotted against the layer's fractional compression, and one can observe that the magnitude of elastic energy stored at the point of (we suppose) the onset of significant interpenetration differed systematically according to the system. When compared at constant fractional compression, it increased in the sequence polymer A, polymer B, and polymer $\mathrm{C}$ and for the homopolymer system was so small that the calculations for this system

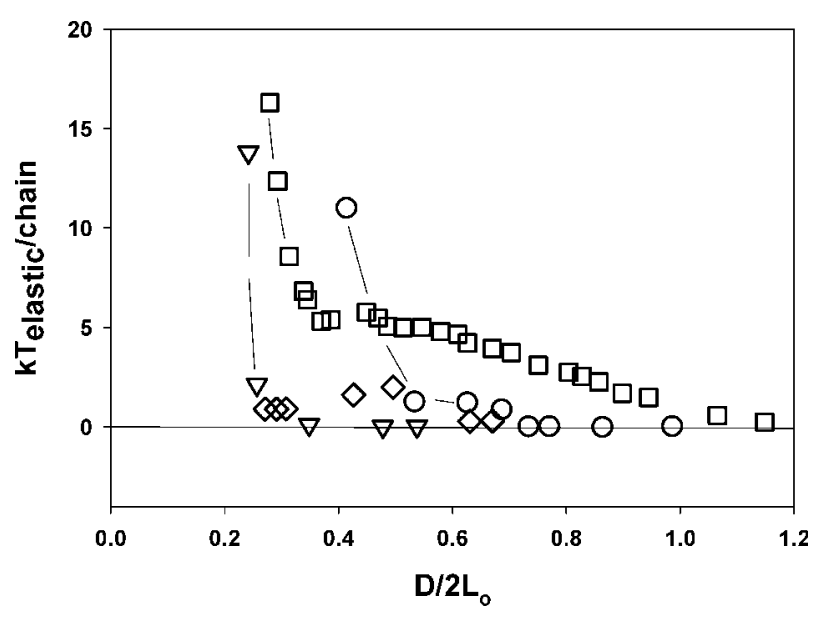

Figure 6. Estimate of the elastic shear energy per polymer chain, with respect to the thermal energy, $k T$, and how this varied with layer compression. The effective storage shear modulus $(1 \mathrm{~Hz})$, normalized to represent the energy stored in units of $k_{\mathrm{B}} T$ per chain, is plotted as a function of the fractional compression, $D / 2 L_{\mathrm{o}}$, for $(\square)$ polymer $\mathrm{C},(\nabla)$ polymer $\mathrm{A},(\bigcirc)$ polymer $\mathrm{B}$, and $(\diamond)$ the adsorbed homopolymer. The main point of this figure is to show that although adsorbed PS homopolymer stored (measured on this scale) an insignificant amount of elastic energy, the endattached chain systems stored considerably more.

were indistinguishable from zero on the scale needed to encompass the other systems. No reason has been advanced at present to explain why, for the end-tethered systems, the normalized stored elastic energy per chain so much exceeded unity. The main point of this figure is to show that whereas adsorbed PS homopolymer stored (measured on this scale) an insignificant amount of elastic energy, the end-attached chain systems stored considerably more.

\section{How Shear and Compressive Forces Were Coupled}

The growing interest in using end-attached polymer layers for friction applications also motivated us to consider these shear forces from the perspective of friction. From this point of view, we defined what we call the small-strain friction coefficient, $\mu$, in analogy to the friction coefficient (the dimensionless ratio of the shear force to the normal load) used to describe kinetic friction. It made no sense to simply take the ratio of shear forces to compressive force because the shear forces, which were measured in the linearresponse regime, were directly proportional to the shear amplitude. The following method was 


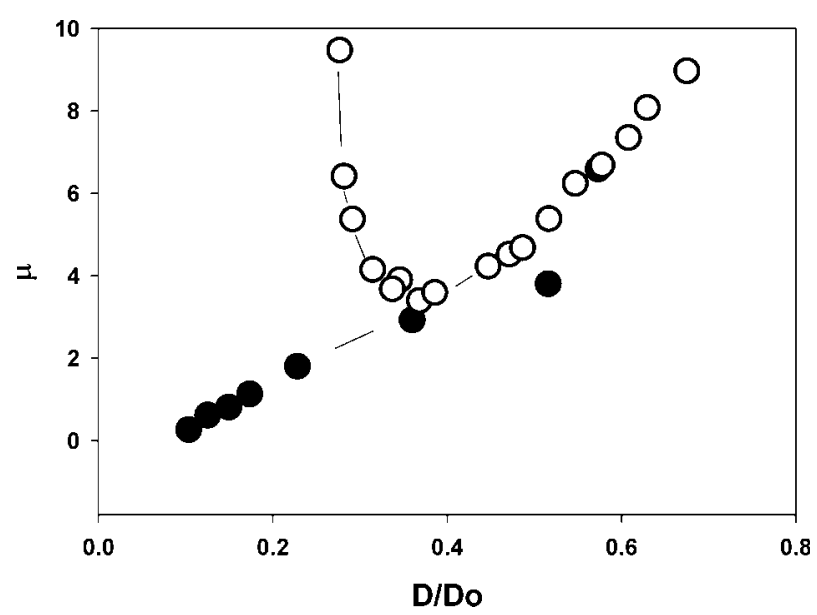

Figure 7. Thickness dependence of the small-amplitude friction coefficient $(\mu)$ for polymer $\mathrm{C}$. This is the dimensionless ratio of the elastic shear modulus $(1 \mathrm{~Hz})$ to the normal pressure derived from data in Figures 1 and 5 as described in the text. On the thickness scale, the actual thickness, $D$, is normalized by the film thickness at the onset of repulsive normal forces, $D_{0}$. The open symbols represent the brushbrush case, and the filled symbols present the brushmica case.

used to normalize them. First, the shear forces were normalized to give the effective shear moduli. Next, the compressive forces were normalized by the effective area of contact (see the Experimental section) to give the normal pressure. With this normalization performed, it was meaningful to compare the dimensionless ratio of the elastic shear modulus to the normal pressure.

$\mu$ is plotted against the dimensionless film thickness in Figure 7; the key point is the comparison between brush-mica and brush-brush situations. There are two striking observations to make. First, the friction coefficient was decidedly not constant; as the film thickness decreased under the action of compressive force, two regimes were observed, corresponding to a small amount and a large amount of compression. $\mu$ at first decreased, indicating that normal forces increased more rapidly than shear forces, but this tendency switched when the compressive forces were high. In this second regime of high compressive force, $\mu$ of the asymmetric brush-mica system continued to decrease monotonically, but for the symmetric brush-brush system, it passed through a minimum and subsequently increased, indicating that for this highly compressed system, the shear forces increased more rapidly than the compressive forces. A similar analysis with respect to the shear frequency at 5 and $52 \mathrm{~Hz}$ led to the same conclusion. ${ }^{20}$

Taken together, these experiments show that the polymer films presented greater elastic resistance to shear than to compression; $\mu$ exceeded unity. The exception was the polymer-mica system at high compression, showing that the chains could slide more readily across a smooth solid surface than against an opposing brush layer with which it could interdigitate.

\section{CONCLUSIONS}

This article presents a comprehensive study of the linear viscoelastic shear response of endattached polymer chains under near-theta-solvent conditions. The main result is to quantify the presence of a shear relaxation time that starts to slow linearly with diminishing thickness $(D)$ at $D \approx L_{\mathrm{o}} / 3$, but the reasons for disagreement with available theories are not understood at this time. Looking to the future, we think that new instrumental technology, developed since this study was undertaken, now enables one to augment measurements of interfacial rheology using optical spectroscopic measurements. Future studies should be capable of probing directly questions of how individual chains fluctuate, using fluorescence methods, ${ }^{41}$ as well as questions of how chains align in confinement, using Raman methods. ${ }^{42}$

The authors are indebted to Hiroshi Watanabe (Kyoto University) for the gift of a polymer sample. They also thank Chih-Yuan Wu and Thuy-Dien Cao (Texas Tech University) for their assistance with figure plotting. For support, the authors are indebted to the U.S. Department of Energy Division of Materials Science (award no. DEFG02-02ER46019) through the Frederick Seitz Materials Research Laboratory at the University of Illinois at Urbana-Champaign. LLD was supported by grant NSF CTS-0500323.

\section{REFERENCES AND NOTES}

1. Ferry, J. D. Viscoelastic Properties of Polymers, 3rd ed.; Wiley: New York, 1980.

2. Hu, H.-W.; Granick, S. Science 1992, 258, 1339.

3. Granick, S.; Hu, H.-W. Langmuir 1994, 10, 3857.

4. Peanasky, J.; Cai, L. L.; Granick, S.; Kessel, C. R. Langmuir 1994, 10, 3874.

5. Cho, K.; Watanabe, H.; Granick, S. J Chem Phys 1999, 110, 9688.

6. Ruths, M.; Granick, S. Tribol Lett 1999, 7, 161. 
7. Parsonage, E.; Tirrell, M.; Watanabe, H.; Nuzzo, R. G. Macromolecules 1991, 24, 1987.

8. Milner, S. T. Science 1991, 251, 905.

9. Szleifer, I.; Carignano, M. A. Adv Chem Phys 1996, 94, 165.

10. Manciu, M.; Ruckenstein, E. Langmuir 2004, 20, 6490.

11. Binder, K. Eur Phys J E 2002, 9, 293.

12. Halperin, A.; Tirrell, M. V.; Lodge, T. P. Adv Polym Sci 1992, 100, 31.

13. Klein, J. Annu Rev Mater Sci 1996, 26, 581.

14. Schorr, P. A.; Kwan, T. C. B.; Kilbey, S. M.; Shaqfeh, E. S. G.; Tirrell, M. Macromolecules 2003, 36, 389.

15. Kreer, T.; Muser, M. H.; Binder, K. Comput Phys Commun 2002, 147, 358.

16. Reiter, G.; Demirel, A. L.; Granick, S. Science 1994, 263, 1741.

17. Peachey, J.; Van Alsten, J.; Granick, S. Rev Sci Instrum 1991, 62, 463.

18. Granick, S.; Demirel, A. L.; Cai, L.; Peanasky, J. Isr J Chem 1995, 35, 75.

19. Dhinojwala, A.; Cai, L.; Granick, S. Langmuir 1996, 12, 4537.

20. Cai, L. L. Ph.D. Thesis, University of Illinois at Urbana-Champaign, 1996.

21. Cai, L. L.; Granick, S. Adv Colloid Interface Sci 2001, 94, 135.

22. Lin, Z. Q.; Granick, S. Langmuir 2003, 19, 7061.

23. Zhu, Y.; Granick, S. Phys Rev Lett 2004, 93, 096101.

24. Tirrell, M.; Parsonage, E.; Watanabe, H.; Dhoot, S. Polym J 1991, 23, 641.

25. Rizzotto, M.; Szleifer, I. J Polym Sci Part B Polym Phys 2005, 43, 1849.
26. Halperin, A.; Alexander, S. Europhys Lett 1988, $6,329$.

27. Grest, G. S. Adv Polym Sci 1999, 138, 149.

28. Lai, P. Y.; Binder, K. J Chem Phys 1993, 98, 2366.

29. Binder, K.; Lai, P. Y.; Wittmer, J. Faraday Discuss 1994, 98, 97.

30. Peters, G. H.; Tildesley, D. J. Phys Rev E 1995, 52, 1882.

31. Doyle, P. S.; Shaqfeh, E. S. G.; Gast, A. P. Phys Rev Lett 1997, 78, 1182.

32. Joanny, J. Langmuir 1992, 8, 989.

33. Rubinstein, M.; Obukhov, S. P. Macromolecules 1993, 26, 1740.

34. Klein, J.; Kamiyama, Y.; Yoshizawa, H.; Israelachvili, J. N.; Fredrickson, G. H.; Pincus, P.; Fetters, L. J. Macromolecules 1993, 26, 5552.

35. Yurasova, T. A.; McLeish, T. C. B.; Semenov, A. N. Macromolecules 1994, 27, 7205.

36. Semenov, A. N. Langmuir 1995, 11, 3560.

37. Semenov, A. N.; Vlassopoulos, D.; Fytas, G.; Vlachos, G.; Fleischer, G.; Roovers, J. Langmuir 1999, $15,358$.

38. de Gennes, P.-G. Scaling Concepts in Polymer Physics; Cornell University Press: Ithaca, NY, 1979.

39. Guzonas, D.; Boils, D.; Hair, M. L. Macromolecules 1991, 24, 3383.

40. Hair, M. L.; Guzonas, D.; Boils, D. Macromolecules 1991, 24, 341.

41. Mukhopadyay, A.; Zhao, J.; Bae, S. C.; Granick, S. Rev Sci Instrum 2003, 74, 3067.

42. Bae, S. C.; Lee, H.; Lin, Z.; Granick, S. Langmuir $2005,13,5685$. 